\title{
Adjuvant chemotherapy in patients with locally advanced bladder cancer after neoadjuvant chemotherapy and radical cystectomy: a systematic review and pooled analysis
}

\author{
Zhiyong Cai", Hang Jin", Jinbo Chen, Jiao Hu, Huihuang Li, Zhenglin Yi, Xiongbing Zu \\ Department of Urology, Xiangya Hospital, Central South University, Changsha, China \\ Contributions: (I) Conception and design: X Zu, Z Cai; (II) Administrative support: X Zu, J Chen; (III) Provision of study materials or patients: H \\ Jin, Z Cai; (IV) Collection and assembly of data: Z Cai, H Jin, J Hu; (V) Data analysis and interpretation: Z Cai, H Jin, J Hu, Z Yi; (VI) Manuscript \\ writing: All authors; (VII) Final approval of manuscript: All authors. \\ \#These authors contributed equally to this work. \\ Correspondence to: Jinbo Chen, Xiongbing Zu. Department of Urology, Xiangya Hospital, Central South University, Changsha 410008, China. Email: \\ chenjinbo1989@yahoo.com, zuxbyyy@126.com.
}

\begin{abstract}
Background: Neoadjuvant chemotherapy (NAC) could ameliorate the stage of locally advanced bladder cancer (LABC) which is defined in pT3/T4 and/or pN+, improve overall survival (OS) before radical cystectomy (RC). However, for LABC, the decision to use adjuvant chemotherapy (AC) after NAC and RC is still controversial.

Methods: We performed a comprehensive search of the PubMed, Embase, and Cochrane Library databases for literature that reported prognosis after using AC following NAC and RC. Cumulative analyses of hazard ratios (HRs) and corresponding 95\% confidence intervals (CIs) were performed. We performed all analyses by Review Manager software, version 5.3, and Stata 15.0.

Results: Six retrospective cohort studies were included, involving 4,346 patients. Pooled analysis results showed that using AC after NAC and RC can improve OS (HR =0.83, 95\% CI: 0.74-0.94, P=0.002; $\mathrm{I}^{2}$ $=0 \%)$ and cancer-specific survival $(\mathrm{CSS})\left(\mathrm{HR}=0.56,95 \% \mathrm{CI}: 0.32-0.99, \mathrm{P}=0.04 ; \mathrm{I}^{2}=0 \%\right)$ but cannot extend recurrence-free survival (RFS) ( $\mathrm{HR}=0.52,95 \% \mathrm{CI}: 0.27-1.01, \mathrm{P}=0.05 ; \mathrm{I}^{2}=53 \%$ ) for $\mathrm{LABC}$ patients.

Conclusions: This pooled analysis shows that AC after NAC and RC can improve the prognosis for patients with LABC.
\end{abstract}

Keywords: Urinary bladder neoplasms; meta-analysis; adjuvant chemotherapy; neoadjuvant chemotherapy (NAC)

Submitted Feb 14, 2020. Accepted for publication Oct 30, 2020.

doi: $10.21037 /$ tau-20-571

View this article at: http://dx.doi.org/10.21037/tau-20-571

\section{Introduction}

Bladder cancer (BCa) is a prevailing, invasive, malignant tumor in the urinary system (1). In 2019, there were 80,470 bladder cancer, with 17,670 deaths, occurred in America (2). Radical cystectomy (RC) is regard as the standard surgery of muscle-invasive bladder cancer (3). With the development of neoadjuvant chemotherapy (NAC), NAC combined with $\mathrm{RC}$ have been increasingly proved effective in $\mathrm{BCa}$, which can improve patients' survival and quality of life. However, most patients will still relapse and develop metastasis (4).
In addition, bladder cancer patients need to monitor tumor recurrence throughout their lives, and full-course treatment of locally advanced bladder cancer (LABC) is so expensive that few patients could afford (5).

Current NAC and radical cystectomy (RC) are recommended by U.S. guidelines as the treatment of choice for LABC (6). NAC can reduce the stage of invasive bladder cancer and improve overall survival (OS) before RC (7). However, for patients with LABC (pT3-T4 or $\mathrm{pN}+$, there is little existing evidence to guide treatment (8). Matthew 
D. Galsky (9) conducted a study involving 5,396 patients with LABC and concluded that there is a significant improvement in overall survival (HR 0.70; 95\% CI: 0.64 to 0.76 ) for those who had adjuvant chemotherapy(AC) after RC. However, the current research on the efficacy of AC after NAC and RC is controversial. Some clinical studies suggest that the use of AC can prolong the survival of patients who received NAC and RC, but some researchers believed that using adjuvant chemotherapy is not beneficial to prognosis $(7,8,10-13)$.

Here, a pooled-analysis was systematically performed to explore whether the prognosis of patients receiving AC after NAC and RC significantly improved.

\section{Methods}

This research was finished in accordance with the Preferred Reporting Items for Systematic Review and Meta-analyses (PRISMA) reporting checklist (available at http://dx.doi. org/10.21037/tau-20-571) (14). Because of the nature of the study design, no ethical standards approval or informed consent was required.

\section{Search strategy}

A comprehensive review of the literatures was performed in accordance with the PRISMA statement in January 2020 in PubMed (ncbi.nlm.nih.gov/pubmed), Embase (embase.com), and the Cochrane Central Search Library (cochranelibrary.com). We used following Search terms to search literature: "Neoadjuvant Therapy"[Mesh] OR (neoadjuvant chemotherapy OR Preoperative Chemotherapy)) AND (neoplasms OR cancer OR carcinoma) AND ("Urinary Bladder Neoplasms"[Mesh] OR (bladder OR urinary bladder)) AND ("Cystectomy"[Mesh] OR Radical Cystectomy) AND ("Chemotherapy, Adjuvant"[Mesh] OR Adjuvant Chemotherapy). We reviewed all abstracts and review studies about this topic and identified manually the references of original studies.

\section{Inclusion/exclusion criteria}

The following selection criteria needed to be met in qualified studies: studies appraising the effect of using AC after neoadjuvant chemotherapy and $\mathrm{RC}$; reports containing important information about $\mathrm{AC}$ use and bladder cancer susceptibility, overall survival (OS), cancer-specific survival (CSS), recurrence-free survival (RFS); sufficient information was provided for a hazard ratio (HR) including a 95\% confidence interval (95\% CI); cohort studies having a controlled group; study's language is English. The following are the exclusion criteria: reviews, case series, case reports, letters, and editorials; studies cannot estimate HR with a 95\% CI; animal-related studies.

\section{Data extraction}

Two independent researchers ( $\mathrm{ZYC}$ and $\mathrm{JH})$ extracted the data of eligible studies. Argument was solved by discussing with a third researcher $(\mathrm{HJ})$. We extracted individually the data from the literature and demographics. The following information were contained: author, ethnicity, area of study, study type, publication year, tumor stage, sample size, age, survival analysis, exposure or intervention definition, adjusted variables, Newcastle-Ottawa Scale (NOS) score, duration and median follow-up. An HR with a $95 \%$ CI was used to assess the association between adjuvant chemotherapy use and OS, CSS, and RFS after neoadjuvant chemotherapy and radical cystectomy. If the HRs, their 95\% CIs and $\mathrm{P}$ values were available, they were obtained from the original articles. If not, we computed the HRs and 95\% CIs according to the methods (15).

\section{Statistical analysis}

We took the HRs and 95\% CIs together to survey the effect of using AC after NAC and radical cystectomy for outcomes. The HRs and 95\% CIs directly were collected in the article if they were available. To avoid calculation errors, two independent researchers completed this process. We tested Statistical heterogeneity in studies by a formal Q-statistic and the chi-squared $\left(\mathrm{I}^{2}\right)$ test. The degree of heterogeneity was investigated through the value of $\mathrm{I}^{2}$ (no heterogeneity: $\mathrm{I}^{2}<25 \%$; moderate heterogeneity: $\mathrm{I}^{2}=25-50 \%$; substantial heterogeneity: $\left.\mathrm{I}^{2}>50 \%\right)$. The random-effects model was utilized when we found substantial heterogeneity. When we found moderate or no heterogeneity, the fixed-effects model was utilized. The level of statistical significance was set at 0.05 (16).We could not assess publication bias $(17,18)$ because the amount of the included studies is small, so to appraise the stability of the pooled-analysis results, a sensitivity analysis was done using the leave-one-out cross validation. Review Manager (RevMan) v5.3 was used to perform this meta-analysis. 

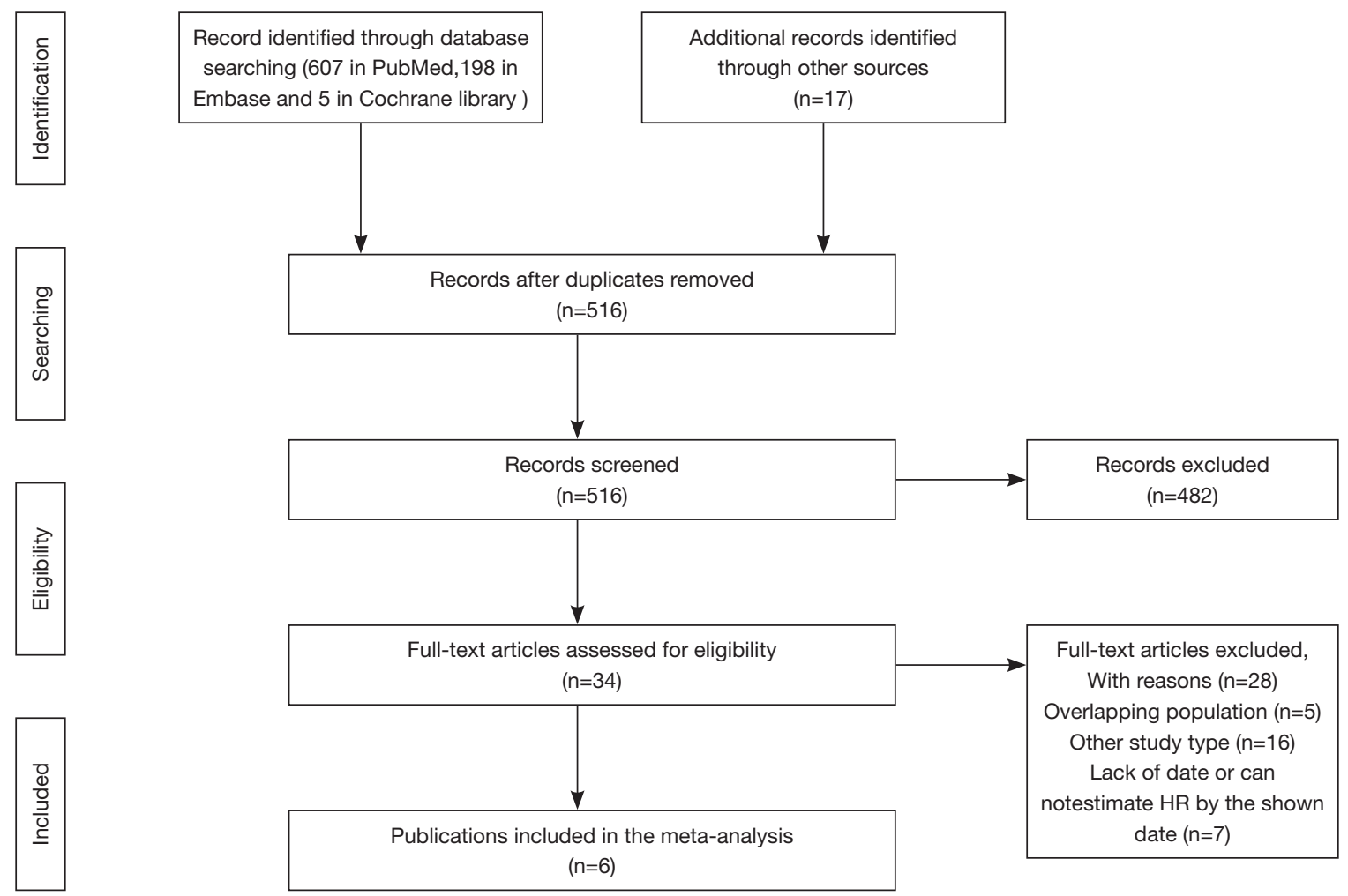

Figure 1 Flow chart of studies selection.

\section{Results}

\section{Study selection and characteristics}

Eventually, we got 607 records through databases. Our present meta-analysis included six studies $(7,8,10-13)$ in accordance with the inclusion and exclusion criteria, which completely investigated the effects of using AC after neoadjuvant chemotherapy and RC and their outcomes (Figure 1). Especially, data were available from four researches on the effects of using adjuvant chemotherapy on the OS of patients with LABC, three studies about RFS, and two studies about CSS. Three thousand ninetysix patients with $\mathrm{pT} 3 / \mathrm{T} 4$ and/or $\mathrm{pN}+$ were included; $76 \%$ $(n=2,355)$ of them were in the exposure group and $24 \%$ $(n=741)$ were in the control group. We summarized the main characteristics of the qualified studies in Table 1. The results are summarized in Table 2. To assess the quality, the Newcastle-Ottawa Scale (NOS) scores (Table S1) of the including studies ranged from 7 to 8 .
$A C$ after neoadjuvant chemotherapy and $R C$ improves $O S$ for $L A B C$

Four studies reported the effects of using AC after neoadjuvant chemotherapy and RC on the OS of patients with $\mathrm{LABC}$, which involved 2,887 patients with $\mathrm{pT} 3 / \mathrm{T} 4$ and/or $\mathrm{pN}+$ \{NAC+RC [2198] vs. NAC+RC+AC [689]\}. A fixed-effects model was utilized because no significant heterogeneity was found between these studies $\left(\mathrm{I}^{2}=0 \%\right.$; $\mathrm{P}=0.50$ ). Mainly, using AC after neoadjuvant chemotherapy and $\mathrm{RC}$ was conducive to the OS of patients with LABC (HR $=0.83$, 95\% CI: 0.74-0.94; $\mathrm{P}=0.002$ ) (Figure 2).

\section{Adjuvant chemotherapy after NAC and RC improves CSS of $L A B C$}

Another two studies showed the association between using AC after neoadjuvant chemotherapy and RC and CSS of patients with bladder cancer, which involved 125 patients with $\mathrm{pT} 3 / \mathrm{T} 4$ and/or $\mathrm{pN}+\{\mathrm{NAC}+\mathrm{RC}[75] v s$. NAC+RC+AC 


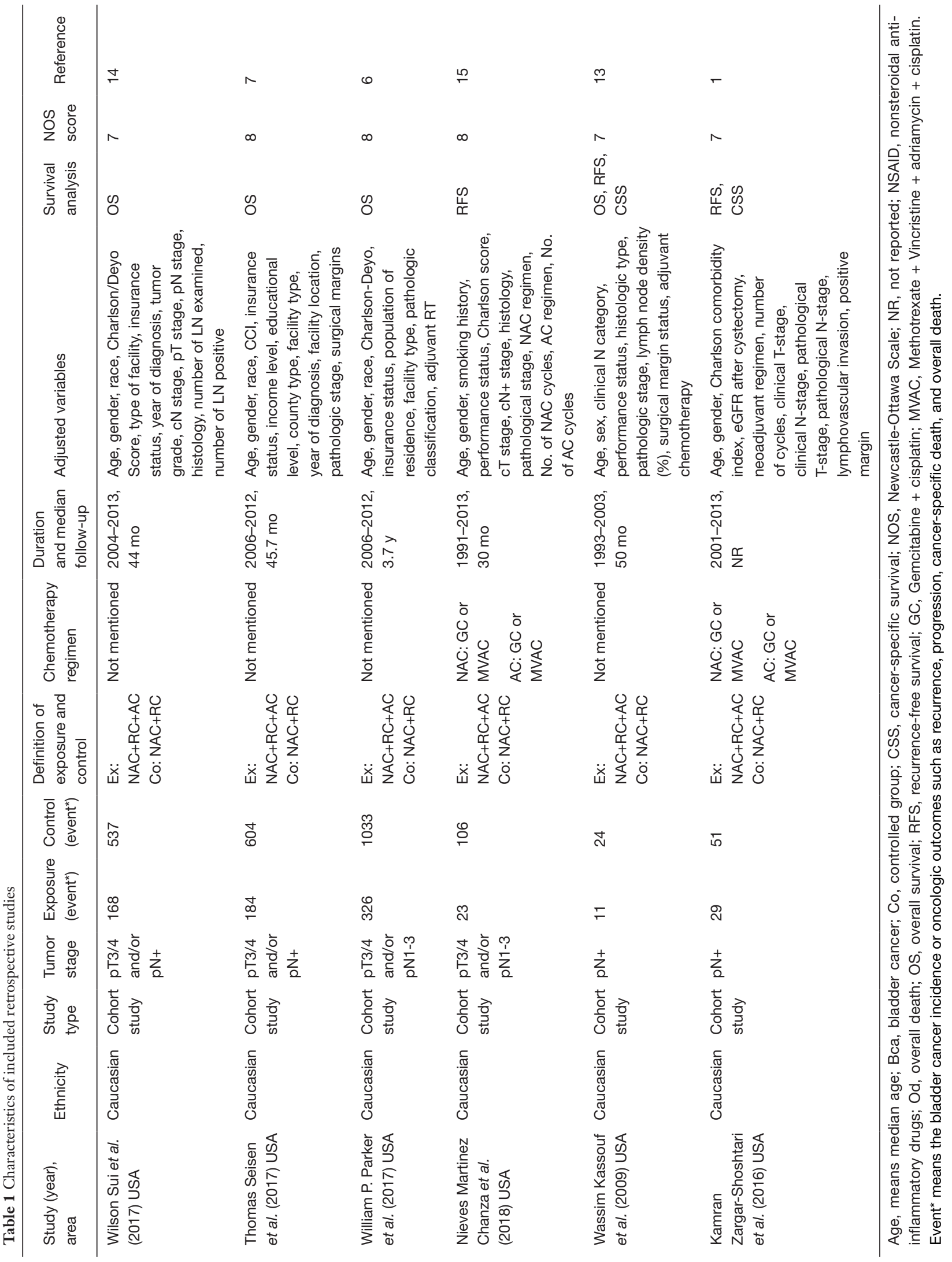


Table 2 Analysis of the effect of using adjuvant chemotherapy after neoadjuvant chemotherapy and radical cystectomy

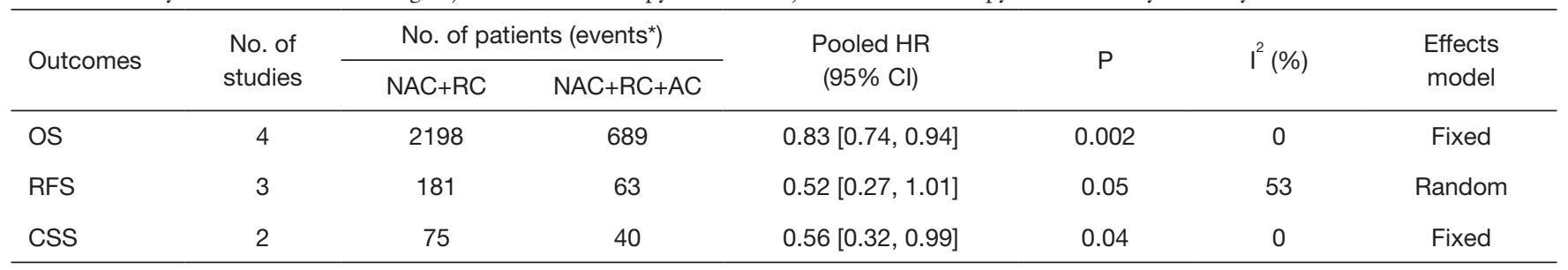

$\mathrm{Cl}$, confidence interval; CSS, cancer-specific survival; HR, hazard ratio; NA, it means that we cannot extract directly or estimate indirectly the number of events from the original article; OS, overall survival; PFS, progression-free survival; RFS, recurrence-free survival. Event* means the bladder cancer incidence or oncologic outcomes such as recurrence, progression, cancer-specific death, and overall death.

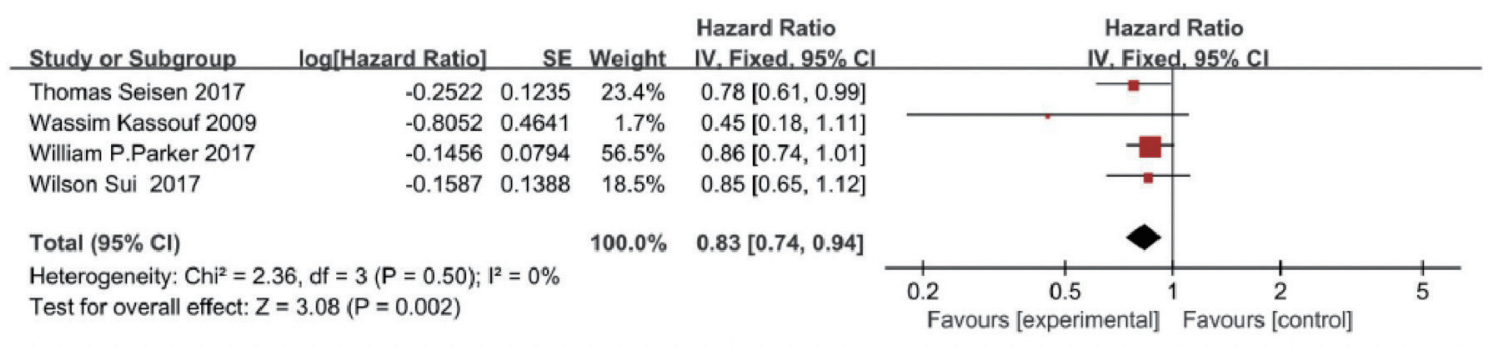

Figure 2 Forest plot of HR for overall survival. Association between using AC after NAC and + RC and overall survival; The diamond indicates the pooled HR value. HR, hazard ratio; CI, confidence interval; SE, standard error.

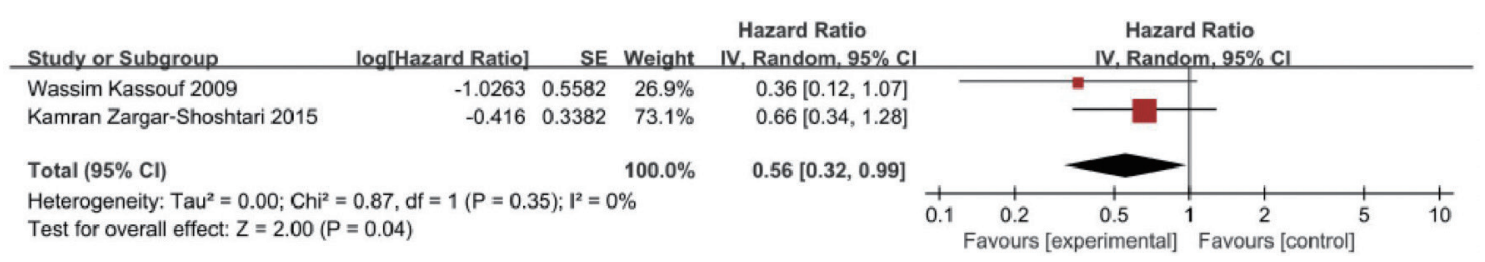

Figure 3 Forest plot of HR for cancer-specific survival. Association between using AC after NAC and +RC and cancer-specific survival; The diamond indicates the pooled HR value. HR, hazard ratio; CI, confidence interval; SE, standard error.

[40]\}. No significant heterogeneity between these studies was found $\left(\mathrm{I}^{2}=0 \% ; \mathrm{P}=0.35\right)$. Overall, using adjuvant chemotherapy after NAC and RC led to increased CSS of the LABC patients ( $\mathrm{HR}=0.56,95 \% \mathrm{CI}$ : $0.32-0.99 ; \mathrm{P}=0.04$ ) (Figure 3).

\section{Adjuvant chemotherapy after NAC and RC marginally affected RFS in LABC}

Three studies recorded the effects of using AC after neoadjuvant chemotherapy and RC on the RFS of bladder cancer patients; 244 patients with $\mathrm{pT} 3 / \mathrm{T} 4$ and/or $\mathrm{pN}+$ were included $\{\mathrm{NAC}+\mathrm{RC}$ [181] vs. NAC+RC+AC [63]\}. A random-effects model was utilized in the analysis because a substantial heterogeneity between the trials was found $\left(\mathrm{I}^{2}\right.$ $=53 \% ; \mathrm{P}=0.12$ ). Mainly, we found no significant relationship between the use of adjuvant chemotherapy after NAC and $\mathrm{RC}$ and the RFS of patients with LABC (HR $=0.52,95 \%$ CI: 0.27-1.01; $\mathrm{P}=0.05$ ) (Figure 4).

\section{Sensitivity analysis and quality of studies}

We performed a sensitivity analysis to evaluate the stability of the pooled-analysis results. Because the overall HR did not change significantly after removing the studies related to heterogeneity, the pooled-analysis was convincing and adequately stable (Figure 5). Two independent researchers assessed the quality of these studies through the NOS 


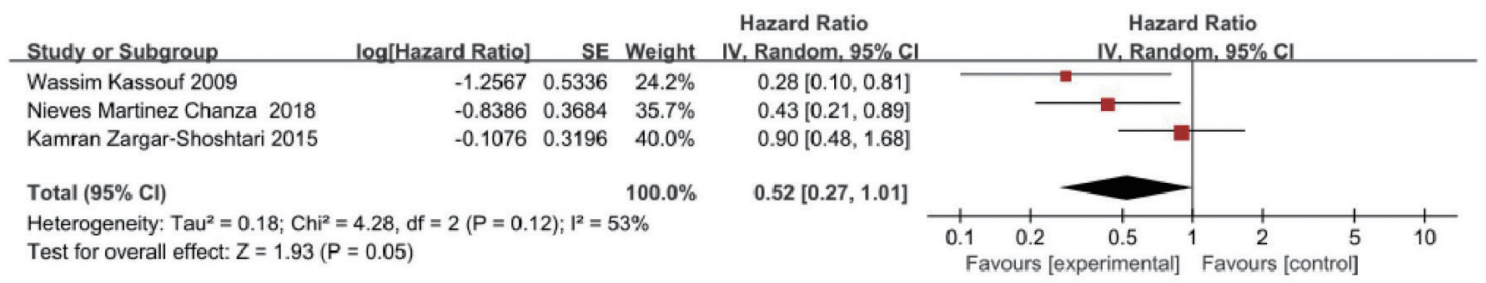

Figure 4 Forest plot of HR for recurrence-specific survival. Association between using AC after NAC and + RC and recurrence-specific survival; The diamond indicates the pooled HR value. HR, hazard ratio; CI, confidence interval; SE, standard error.

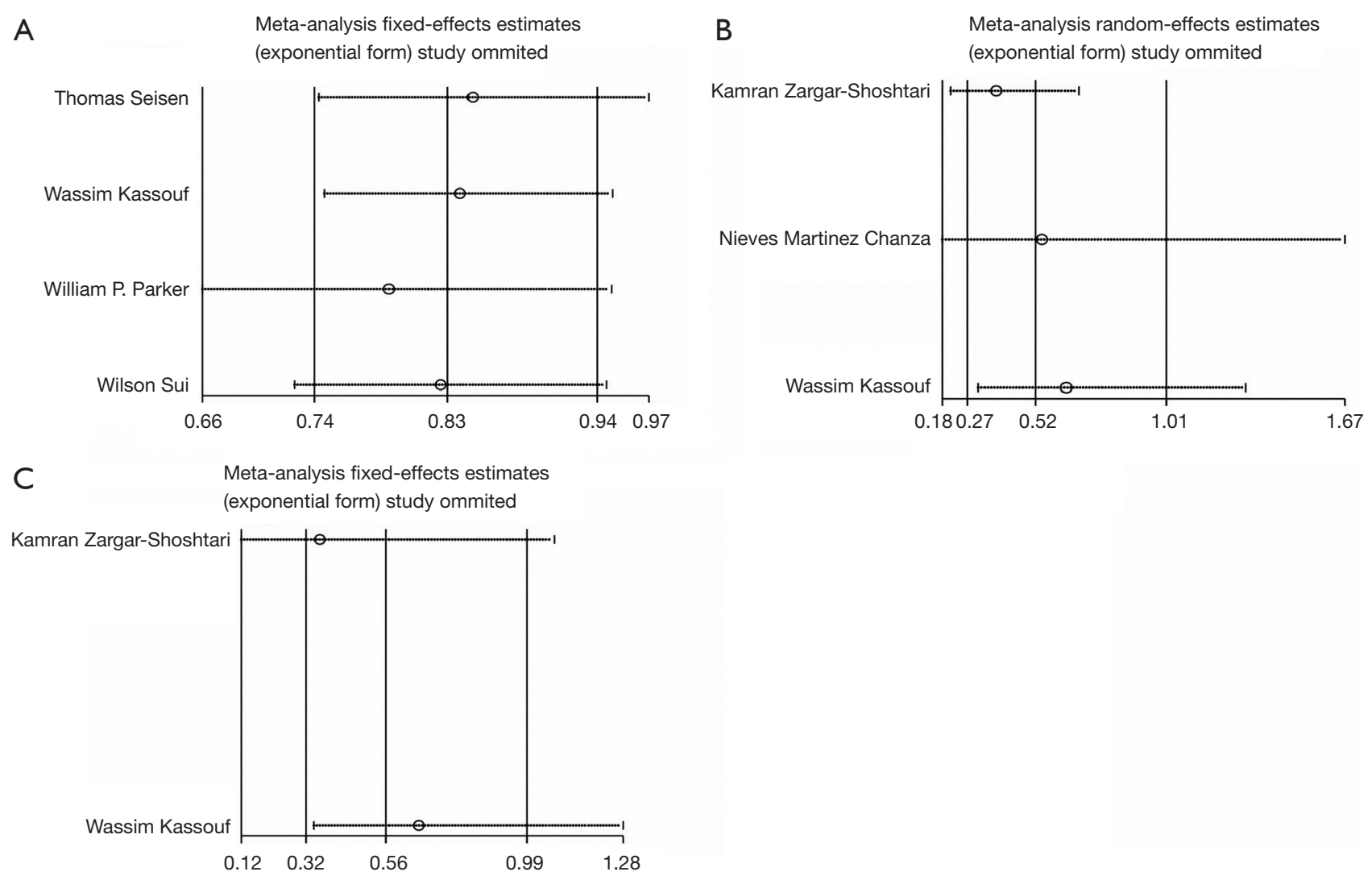

Figure 5 Sensitivity analysis for (A) overall survival, (B) recurrence-free survival, (C) cancer-specific survival.

(Newcastle-Ottawa Scale) (16). High-quality studies: scores of 7 to 9 ; low-quality study: score of $<7$.

\section{Discussion}

Patients with LABC after RC has a poor five-year OS of 25-38\% (19-21). Despite treatment with NAC, patients having pathologic lymph node involvement also have a poor median OS of 13-26 months (22-24). These patients are limited to utilizing additional chemotherapy for treatment.
Bryan M. Burt (25) reported that AC after neoadjuvant chemotherapy and esophagectomy provided a benefit to the OS of patients with esophageal cancer. In addition, Haiying Sun et al. (26) had the same result for cervical cancer, so we wanted to know whether the same regimen was effective for patients with LABC and whether it could improve their prognosis. Thomas Seisen et al. reported that AC after neoadjuvant chemotherapy and $\mathrm{RC}$ was related to an OS benefit for patients with pT3/T4 and/or pN+ bladder cancer (12), but whether patients with LABC after NAC 
and RC benefited from further adjuvant chemotherapy was unclear, although many scholars support additional chemotherapy (27).

Six retrospective cohort studies investigated the effects of using additional adjuvant chemotherapy after NAC and RC on the OS, RFS, and CSS of LABC. In the study by Wilson Sui (13), the AC group had a longer median OS compared with the observation group ( 23 vs. 20 months), but the result was not statistically significant, and AC was not related to the risk of death in a multivariate analysis. This conclusion was also supported by W.P. Parker (8) and W. Kassouf (10) Conversely, Seison (12) reported that the AC group had a significantly longer OS than the observation group. There was also a disagreement about the CSS of the AC group between K. Zargar-Shoshtari (7), who concluded that $\mathrm{AC}$ was not associated with obvious improvement in CSS, and W. Kassouf (10), whose study presented a positive result, proposing that $\mathrm{LABC}$ patients receiving AC after NAC and RC could realize an improved CSS. W. Kassouf (10) and N. Martinez Chanza (11) supported the view that AC could improve the RFS of the patients, and K. ZargarShoshtari (7) reported that RFS which was not statistically significant appeared longer in the experimental group. The current pooled-analysis showed a significant association between using additional adjuvant chemotherapy after NAC and RC and the CSS and OS of patients with bladder carcinoma, but no effect on the RFS. Because the data provided by these six studies was not very comprehensive, larger prospective studies need to verify the outcomes.

Actually, this present study represents the first metaanalysis of an effectiveness evaluation of AC after neoadjuvant chemotherapy and RC. Indeed, 3096 patients were included in these six studies with $\mathrm{pT} 3 / \mathrm{T} 4$ and/or $\mathrm{pN}+$ disease after NAC and RC. 741 of them further used AC that was independently related to overall survival and CSS. The results concluded that using additional AC after neoadjuvant chemotherapy and $\mathrm{RC}$ could extend the OS and CSS of patients with pT3/T4 and/or pN+ BCa but added no obvious benefit to their RFS.

Node-positive bladder cancer was a type of LABC which means poor prognosis. As the limitation of pooled data, we could not conduct subgroup analysis on $\mathrm{pN}$ - or $\mathrm{pN}+$ about OS or CSS for additional AC. Walz et al. considered that either MVAC or GC had no significant effect on CSS or OS after RC in high-risk patients which included positive node patients (28). However, Pak et al. argued that AC after $\mathrm{RC}$ was associated with improved OS in patients with nodepositive bladder cancer (29). Consequently, the effect of additional AC on the node-positive patients is still unclear.

In aspect of chemotherapy regimen of NAC and AC, not only merely two of six studies described the exact chemotherapy regimens of patients, but also all of them didn't mentioned whether same regimen used in NAC and AC. In EAU guideline, they strongly recommended to offer cisplatin-based combination chemotherapy to LABC in NAC and AC (30). However, we didn't find any studies to identify the same chemotherapy regimen in NAC and AC.

Furthermore, the results didn't show whether all of LABC patients need to have an additional AC. However, there have been some studies indicated patients with different biomarkers had different sensitivity on chemotherapy. Woonyoung Choi et al. found that $\mathrm{p} 53$ like MIBCs were consistently resistant to neoadjuvant methotrexate, vinblastine, doxorubicin and cisplatin chemotherapy (31). Van Allen et al. found that Somatic ERCC2 mutations correlate with complete response to cisplatin-based chemosensitivity in MIBC (32). Consequently, we should stratify patients with different biomarkers.

Different from chemotherapy only era, with the rise of immunotherapy, more and more immunotherapy agents such as PD-1 blockade and PD-L1 blockade have been applied in therapy of LABC. Necchi et al. found that Median OS for those continuing atezolizumab (PD-L1 blockade) treatment after the previous platinum-based chemotherapy was 12.8 months, compared to 3.6 months for those not treated with atezolizumab (33). In another clinical trail-Keynote045, comparing pembrolizumab (blockade interaction between PD-1 and PD-L1) to chemotherapy after previously platinum-based chemotherapy. The median OS was 10.3 vs. 7.4 months (34). These studies showed that application of immunotherapy agents followed by NAC and RC probably have a better prognosis.

Some limitations also exist in our meta-analysis. First, the type of chemotherapy was not available, because these six studies used different chemotherapies. Some studies used platinum-based regimens, but some used carboplatinbased regimens, which probably resulted in different effects for patients. Second, although we confirmed the positive effects of using additional AC after neoadjuvant chemotherapy and RC for patients with pT3/T4 and/or $\mathrm{pN}+$, we do not know whether using additional AC after neoadjuvant chemotherapy and $\mathrm{RC}$ could extend the OS or CSS of patients with pT0-2 bladder cancer, because these six studies selected only patients with pT3/T4 and/ or $\mathrm{pN}+$ cancer. Third, English is the only language of these 
qualified studies, so increased publication bias might be found in the exclusion of studies in other languages.

\section{Conclusions}

Our meta-analysis supported a favorable clinical role for additional AC for patients with LABC after neoadjuvant chemotherapy and RC. We found that using additional AC after neoadjuvant chemotherapy and RC could extend the OS and CSS of patients with LABC. Large, prospective, multicenter, randomized controlled trials are still essential to confirm the role of AC for LABC.

\section{Acknowledgments}

Funding: This work was supported by the National Natural Science Foundation of China (81902592), Hunan Province Key R\&D Program (2019SK2202) and Xiangya Hospital Youth Fund (2018Q09).

\section{Footnote}

Reporting Checklist: The authors have completed the PRISMA reporting checklist. Available at http://dx.doi. org/10.21037/tau-20-571

Conflicts of Interest: All authors have completed the ICMJE uniform disclosure form (available at http://dx.doi. org/10.21037/tau-20-571). Dr. XZ serves as an unpaid editorial board member of Translational Andrology and Urology from Mar 2015 to Feb 2021. The other authors have no conflicts of interest to declare.

Ethical Statement: The authors are accountable for all aspects of the work in ensuring that questions related to the accuracy or integrity of any part of the work are appropriately investigated and resolved.

Open Access Statement: This is an Open Access article distributed in accordance with the Creative Commons Attribution-NonCommercial-NoDerivs 4.0 International License (CC BY-NC-ND 4.0), which permits the noncommercial replication and distribution of the article with the strict proviso that no changes or edits are made and the original work is properly cited (including links to both the formal publication through the relevant DOI and the license). See: https://creativecommons.org/licenses/by-nc-nd/4.0/.

\section{References}

1. Herr HW. Transurethral resection of muscle-invasive bladder cancer: 10-year outcome. J Clin Oncol 2001;19:89-93.

2. Siegel RL, Miller KD, Jemal A. Cancer statistics, 2019. CA Cancer J Clin 2019;69:7-34.

3. Tong $\mathrm{S}$, Yang $\mathrm{Z}, \mathrm{Zu} \mathrm{X}$, et al. Anterior versus posterior approach laparoscopic radical cystectomy: a retrospective analysis. World J Surg Oncol 2019;17:9.

4. Sylvester RJ, van der Meijden AP, Oosterlinck W, et al. Predicting recurrence and progression in individual patients with stage Ta T1 bladder cancer using EORTC risk tables: a combined analysis of 2596 patients from seven EORTC trials. Eur Urol 2006;49:466-5; discussion 475-7.

5. Sievert KD, Amend B, Nagele U, et al. Economic aspects of bladder cancer: what are the benefits and costs? World J Urol 2009;27:295-300.

6. Clark PE, Spiess PE, Agarwal N, et al. NCCN Guidelines Insights: Bladder Cancer, Version 2.2016. J Natl Compr Canc Netw 2016;14:1213-24.

7. Zargar-Shoshtari K, Kongnyuy M, Sharma P, et al. Clinical role of additional adjuvant chemotherapy in patients with locally advanced urothelial carcinoma following neoadjuvant chemotherapy and cystectomy. World J Urol 2016;34:1567-73.

8. Parker WP, Habermann EB, Day CN, et al. Adverse Pathology After Neoadjuvant Chemotherapy and Radical Cystectomy: The Role of Adjuvant Chemotherapy. Clin Genitourin Cancer 2017;S1558-7673(17)30207-0.

9. Galsky MD, Stensland KD, Moshier E, et al. Effectiveness of Adjuvant Chemotherapy for Locally Advanced Bladder Cancer. J Clin Oncol 2016;34:825-32.

10. Kassouf W, Agarwal PK, Grossman HB, et al. Outcome of patients with bladder cancer with $\mathrm{pN}+$ disease after preoperative chemotherapy and radical cystectomy. Urology 2009;73:147-52.

11. Martinez Chanza N, Werner L, Plimack E, et al. Incidence, Patterns, and Outcomes with Adjuvant Chemotherapy for Residual Disease After Neoadjuvant Chemotherapy in Muscle-invasive Urinary Tract Cancers. Eur Urol Oncol 2020;3:671-9.

12. Seisen T, Jamzadeh A, Leow JJ, et al. Adjuvant Chemotherapy vs Observation for Patients With Adverse Pathologic Features at Radical Cystectomy Previously Treated With Neoadjuvant Chemotherapy. JAMA Oncol 2018;4:225-9. 
13. Sui W, Lim EA, Joel Decastro G, et al. Use of Adjuvant Chemotherapy in Patients with Advanced Bladder Cancer after Neoadjuvant Chemotherapy. Bladder Cancer 2017;3:181-9.

14. Moher D, Liberati A, Tetzlaff J, et al. Preferred reporting items for systematic reviews and meta-analyses: the PRISMA statement. Ann Intern Med 2009;151:264-9, w64.

15. Tierney JF, Stewart LA, Ghersi D, et al. Practical methods for incorporating summary time-to-event data into metaanalysis. Trials 2007;8:16.

16. Higgins JP, Thompson SG, Deeks JJ, et al. Measuring inconsistency in meta-analyses. Bmj 2003;327:557-60.

17. Begg CB, Mazumdar M. Operating characteristics of a rank correlation test for publication bias. Biometrics 1994;50:1088-101.

18. Egger M, Davey Smith G, Schneider M, et al. Bias in meta-analysis detected by a simple, graphical test. Eur Urol 2007;51:199-203; discussion 204-6.

19. Abdel-Latif M, Abol-Enein H, El-Baz M, et al. Nodal involvement in bladder cancer cases treated with radical cystectomy: incidence and prognosis. J Urol 2004;172:85-9.

20. Manoharan M, Katkoori D, Kishore TA, et al. Outcome after radical cystectomy in patients with clinical $\mathrm{T} 2$ bladder cancer in whom neoadjuvant chemotherapy has failed. BJU Int 2009;104:1646-9.

21. Stein JP, Cai J, Groshen S, et al. Risk factors for patients with pelvic lymph node metastases following radical cystectomy with en bloc pelvic lymphadenectomy: concept of lymph node density. J Urol 2003;170:35-41.

22. Iyer G, Tully CM, Zabor EC, et al. Neoadjuvant Gemcitabine-Cisplatin Plus Radical Cystectomy-Pelvic Lymph Node Dissection for Muscle-invasive Bladder Cancer: A 12-year Experience. Clin Genitourin Cancer 2020;18:387-94.

23. Sonpavde G, Goldman BH, Speights VO, et al. Quality of pathologic response and surgery correlate with survival for patients with completely resected bladder cancer after neoadjuvant chemotherapy. Cancer 2009;115:4104-9.

24. Zargar H, Zargar-Shoshtari K, Lotan Y, et al. Final Pathological Stage after Neoadjuvant Chemotherapy and Radical Cystectomy for Bladder Cancer-Does pT0 Predict Better Survival than pTa/Tis/T1? J Urol 2016;195:886-93.

25. Burt BM, Groth SS, Sada YH, et al. Utility of Adjuvant Chemotherapy After Neoadjuvant Chemoradiation and Esophagectomy for Esophageal Cancer. Ann Surg 2017;266:297-304.

26. Sun H, Huang K, Tang F, et al. Adjuvant chemotherapy after surgery can improve clinical outcomes for patients with IB2-IIB cervical cancer with neoadjuvant chemotherapy followed by radical surgery. Sci Rep 2018;8:6443.

27. Tan WS, Lamb BW, Payne H, et al. Management of Node-Positive Bladder Cancer After Neoadjuvant Chemotherapy and Radical Cystectomy: A Survey of Current UK Practice. Clin Genitourin Cancer 2015;13:e153-8.

28. Walz J, Shariat SF, Suardi N, et al. Adjuvant chemotherapy for bladder cancer does not alter cancer-specific survival after cystectomy in a matched case-control study. BJU Int 2008;101:1356-61.

29. Pak S, You D, Jeong IG, et al. Adjuvant chemotherapy versus observation after radical cystectomy in patients with node-positive bladder cancer. Sci Rep 2019;9:8305.

30. Witjes JA, Bruins HM, Cathomas R, et al. European Association of Urology Guidelines on Muscle-invasive and Metastatic Bladder Cancer: Summary of the 2020 Guidelines. Eur Urol 2021;79:82-104.

31. Choi W, Porten S, Kim S, et al. Identification of distinct basal and luminal subtypes of muscle-invasive bladder cancer with different sensitivities to frontline chemotherapy. Cancer Cell 2014;25:152-65.

32. Van Allen EM, Mouw KW, Kim P, et al. Somatic ERCC2 mutations correlate with cisplatin sensitivity in muscle-invasive urothelial carcinoma. Cancer Discov 2014;4:1140-53.

33. Necchi A, Joseph RW, Loriot Y, et al. Atezolizumab in platinum-treated locally advanced or metastatic urothelial carcinoma: post-progression outcomes from the phase II IMvigor210 study. Ann Oncol 2017;28:3044-50.

34. Bellmunt J, de Wit R, Vaughn DJ, et al. Pembrolizumab as Second-Line Therapy for Advanced Urothelial Carcinoma. N Engl J Med 2017;376:1015-26.

Cite this article as: Cai Z, Jin H, Chen J, Hu J, Li H, Yi Z, $\mathrm{Zu} \mathrm{X}$. Adjuvant chemotherapy in patients with locally advanced bladder cancer after neoadjuvant chemotherapy and radical cystectomy: a systematic review and pooled analysis. Transl Androl Urol 2021;10(1):283-291. doi: 10.21037/tau-20-571 
Table S1 The Newcastle-Ottawa Scale (NOS) for assessing the quality of cohort studies

\begin{tabular}{|c|c|c|c|c|c|c|c|c|c|c|}
\hline \multirow[b]{2}{*}{ Study (year) } & \multicolumn{4}{|c|}{ Selection } & \multicolumn{2}{|c|}{ Comparability } & \multicolumn{3}{|c|}{ Assessment of outcome } & \multirow{2}{*}{$\begin{array}{l}\text { Total quality } \\
\text { score }\end{array}$} \\
\hline & $\begin{array}{l}\text { Representative-ness of } \\
\text { the exposed cohort }\end{array}$ & $\begin{array}{l}\text { Ascertainment of } \\
\text { exposure }\end{array}$ & $\begin{array}{l}\text { Selection of } \\
\text { Controls }\end{array}$ & $\begin{array}{l}\text { Demonstration that outcome of interest } \\
\text { was not present at start of study }\end{array}$ & $\begin{array}{l}\text { Study controls for the } \\
\text { most important factors }\end{array}$ & $\begin{array}{l}\text { Study controls for any } \\
\text { additional factor }\end{array}$ & $\begin{array}{l}\text { Assessment of } \\
\text { outcome }\end{array}$ & $\begin{array}{l}\text { Was follow-up long enough } \\
\text { for outcomes to occur }\end{array}$ & $\begin{array}{l}\text { Adequacy of follow up } \\
\text { of cohort }\end{array}$ & \\
\hline Wilson Sui (2017) & * & * & * & - & * & * & * & * & - & 7 \\
\hline Thomas Seisen (2017) & * & * & * & - & * & * & * & * & * & 8 \\
\hline William P. Parker (2017) & * & * & * & - & * & * & * & * & * & 8 \\
\hline Nieves Martinez Chanza (2018) & * & * & * & - & * & * & * & * & * & 8 \\
\hline Wassim Kassouf (2009) & * & * & * & - & * & * & * & * & - & 7 \\
\hline Kamran Zargar-Shoshtari (2016) & * & * & * & - & * & * & * & * & - & 7 \\
\hline
\end{tabular}

